\title{
Gestão de projetos acadêmicos e competências comportamentais na educação
}

\section{profissional e tecnológica}

\author{
Management of academic projects and behavioral skills in professional and technological education \\ Gestión de proyectos académicos y habilidades conductuales en la educación profesional y
}

tecnológica

Recebido: 09/12/2021 | Revisado: 14/12/2021 | Aceito: 17/12/2021 | Publicado: 02/01/2022

\author{
Marcos Canto Machado \\ ORCID: https://orcid.org/0000-0003-2048-8843 \\ Fundação Indaiatubana de Educação e Cultura, Brasil \\ E-mail: marcos.machado@fiec.edu.br \\ Jessica Suárez Campoli \\ ORCID: https://orcid.org/0000-0003-0917-5109 \\ Universidade de São Paulo, Brasil \\ E-mail: jessica.campoli@usp.br
}

\begin{abstract}
Resumo
A educação profissional e tecnológica possui a premissa legal de atrelar aspectos teóricos e práticos e a importância do trabalho como base educativa, inserindo ativamente o aluno em situações que são necessárias diversas competências. Entretanto, muitos dos projetos desenvolvidos nesta modalidade educacional pouco contribuem para estes aspectos ao não propiciar condições para desenvolvimento destas competências comportamentais ("soft-skills") importantes para a iniciação, planejamento, execução, monitoramento, controle e encerramento dos projetos. Deste modo, este trabalho objetivou inserir estratégias gerenciais baseadas no guia PMBOK para o desenvolvimento dos trabalhos de conclusão de um curso superior em tecnologia. A elaboração dos TCCs iniciou-se com uma sessão de "Design thinking" como dinâmica motivadora e ao longo das demais etapas houve a necessidade de utilização de ferramentas como análise SWOT, modelo de negócios Canvas, metodologia Kanban, diagrama de Gantt, entre outras. Além disto, houveram etapas de verificação através de questionários que indicaram a importância dos projetos educacionais ao simular situações do futuro exercício profissional e também relacionaram positivamente e negativamente competências como comunicação, flexibilidade, integridade, habilidades interpessoais, atitude positiva, profissionalismo, responsabilidade, trabalho em equipe, ética e aptidão técnica, através de análise de agrupamentos por similaridade. Por fim, ressaltaramse os aspectos benéficos desta estratégia ao superar uma formação majoritariamente técnica, mas integrada ao desenvolvimento profissional e humano.
\end{abstract}

Palavras-chave: PMBOK; Soft-skills; Ensino superior; Design thinking; Metodologias ativas.

\begin{abstract}
Professional and technological education has the legal premise of linking theoretical and practical aspects and the importance of work as an educational basis, actively inserting the student in situations that require different skills. However, many of the projects developed in this educational modality contribute little to these aspects by not providing conditions for the development of these behavioral skills (soft-skills) that are important for the initiation, planning, execution, monitoring, control and closure of projects. Thus, this work aimed to insert managerial strategies based on the PMBOK guide for the development of completion works (TCCs) of a higher education course in technology. The elaboration of the TCCs began with a "Design thinking" session as a motivating dynamic and throughout the other stages there was a need to use tools such as SWOT analysis, Canvas business model, Kanban methodology, Gantt diagram, among others. In addition, there were verification steps through questionnaires that indicated the importance of educational projects when simulating situations of future professional practice and also positively and negatively related skills such as communication, flexibility, integrity, interpersonal skills, positive attitude, professionalism, responsibility, work in team, ethics and technical aptitude, through the analysis of clusters by similarity. Finally, the beneficial aspects of this strategy were highlighted, as it went beyond a mostly technical training, but integrated with professional and human development.
\end{abstract}

Keywords: PMBOK; Soft-skills; University education; Design thinking; Active methodologies.

\section{Resumen}

La educación profesional y tecnológica tiene como premisa legal vincular los aspectos teóricos y prácticos y la importancia del trabajo como base educativa, insertando activamente al alumno en situaciones que requieran 
competencias diferentes. Sin embargo, muchos de los proyectos desarrollados en esta modalidad educativa aportan poco a estos aspectos al no brindar condiciones para el desarrollo de estas habilidades conductuales (soft-skills) que son importantes para el inicio, planificación, ejecución, seguimiento, control y cierre. de proyectos. Así, este trabajo tuvo como objetivo insertar estrategias gerenciales basadas en la guía del PMBOK para el desarrollo de trabajos de terminación (TCCs) de un curso de educación superior en tecnología. La elaboración de los TCCs se inició con una sesión de Design thinking como dinámica motivadora y a lo largo de las demás etapas hubo necesidad de utilizar herramientas como análisis, modelo de negocio Canvas, metodología Kanban, diagrama de Gantt, entre otras. Además, hubo pasos de verificación a través de cuestionarios que indicaron la importancia de los proyectos educativos a la hora de simular situaciones de futura práctica profesional y también relacionadas positiva y negativamente habilidades como comunicación, flexibilidad, integridad, habilidades interpersonales, actitud positiva, profesionalismo, responsabilidad, trabajo en equipo, ética y aptitud técnica, mediante el análisis de clusters por similitud. Finalmente, se destacaron los aspectos beneficiosos de esta estrategia, que fue más allá de una formación mayoritariamente técnica, pero integrada con el desarrollo profesional y humano.

Palabras clave: PMBOK; Soft-skills; Enseñanza superior; Design thinking; Metodologías activas.

\section{Introdução}

Os projetos de acordo com o "Project Management Institute" [PMI] podem ser entendidos como empreendimentos com uma duração delimitada e aplicado às mais diversas áreas a fim de suprir demandas por produtos e serviços (PMI, 2017). Estas definições podem ser similarmente aplicadas no desenvolvimento de projetos educacionais que possibilitam inúmeros benefícios nos processos de ensino e aprendizagem dos alunos (Grabowski, 2014; Parolin, 2008). Neste cenário, ações voltadas a gestão de projetos aplicadas à Educação Profissional e Tecnológica (EPT) permitem a aprendizagem do saber fazer uma vez que, envolvido no processo de produzir, o aluno é estimulado a questionar, pesquisar e procurar novas soluções para as problemáticas encontradas (Bancino \& Zevalkink, 2007; DeLuiz, 2001).

Para tal, os cursos orientados à EPT devem oportunizar situações em que os alunos podem aliar os conteúdos curriculares à técnica, aproximando-os de situações que requerem a elaboração de testes para hipóteses, responder questões e solucionar problemas (Ritter, et al., 2018; Parolin, 2008) e participando ativamente do processo e desenvolvem a capacidade de pensar, aprender e aplicar (Almeida, 2002). Isto deve-se pois naturalmente os projetos mobilizam competências cognitivas, estimulam o engajamento, trabalho em equipe, planejamento estratégico, controle de tempo, análise de risco, autonomia, etc. Estas são competências profissionais bastante importantes e devem ser relacionadas aos currículos educacionais propondo-se a incorporar o dinamismo e complexidade do ambiente de trabalho (Ritter, et al., 2018; Laker \& Powell, 2011; Parolin, 2008; DeLuiz, 2001). Além disto, Maximiano (2019) destaca a necessidade de competências gerenciais técnicas, humanas e conceituais voltadas, por exemplo, à liderança, tomada de decisão, mapeamento de processos e avaliação de projetos.

Além disto, tais ações contribuem para o desenvolvimento de outras competências e qualidades comportamentais denominadas "soft skills" como as atitudes positivas, planejamento e organização, comunicação, pensamento crítico e ser capaz de trabalhar em equipe, todas alinhadas e necessárias ao (futuro) exercício profissional (Maximiano, 2019; Ritter, et al., 2018; Robles, 2012). As "soft skills" em uma perspectiva do desenvolvimento interpessoal diferenciam-se das "hard skills" que são expertises desenvolvidas tecnicamente e tangíveis quanto a avaliação do seu aprendizado como por exemplo a utilização de um equipamento ou software, aprendizagem de uma metodologia de trabalho, fazer cálculos, etc. (Laker \& Powell, 2011; Andrews \& Higson, 2010).

No que se refere ao desenvolvimento técnico e a gestão de projetos, podem ser utilizadas estratégias gerenciais e práticas do guia "Project Management Book of Knowledge" [PMBOK]. Devido a sua importância, o guia PMBOK tornou-se um padrão basal para a maioria das metodologias existentes pois identifica e conceitua processos, áreas de conhecimento, ferramentas e técnicas orientadas para as etapas de iniciação, planejamento, execução, monitoramento e controle e encerramento, onde a gestão de projetos é realizada por meio da aplicação e integração destas etapas (PMI, 2017). Para tal, diversas ferramentas gerenciais podem ser utilizadas para iniciar os projetos (e.g. termo de abertura e estrutura analítica do projeto), subsidiar decisões (e.g. 
Matriz SWOT), planejar entregas (e.g. diagrama de Gantt e sistema Kanban) e conferir uma visão estratégica do projeto (e.g. modelo de negócios Canvas).

As práticas estabelecidas no PMBOK e ferramentas gerenciais podem ser aplicadas à diversas situações sendo útil e embasando a tríade aluno-escola-professor na formação para o trabalho e traduzindo para a EPT as diversas competências elencadas anteriormente (Parolin, 2008). Além disto, tal entendimento permite exequir as disposições da Lei $\mathrm{n}^{\circ}$ decreto $\mathrm{n}^{\circ} 5154$ de 2004 que regulamenta os artigos da Lei no 9394 (Diretrizes e Bases da Educação Nacional) relacionados à EPT, tais como a importância do trabalho como base educativa e que teoria e prática são indissociáveis.

Destarte, este trabalho objetivou inserir estratégias gerenciais baseadas no guia PMBOK ao longo do trabalho de conclusão [TCCs] de um curso superior de tecnologia contribuindo para que que os alunos analisem e articulem o conhecimento teórico com aspectos práticos no desenvolvimento de projetos. Além disso, indiretamente procurou-se analisar e desenvolver competências necessárias a prática profissional.

\section{Metodologia}

Utilizou-se uma abordagem qualitativa e aplicada para o desenvolvimento da pesquisa (Gerhardt \& Silveira, 2009). Para tal, explorou-se o estudo de caso da implementação de ferramentas gerenciais e estratégias apresentadas no PMBOK ao longo do desenvolvimento dos trabalhos de conclusão de curso (TCCs) de 25 alunos de um curso superior de tecnologia em processos químicos de uma instituição municipal de ensino. A elaboração dos TCCs ocorreu ao longo de 36 semanas distribuídas em dois semestres letivos, inseridos em uma disciplina de projetos industriais com 4 horas de carga semanal.

Inicialmente, antes mesmo da delimitação dos temas dos TCCs, foi realizada uma dinâmica utilizando os conceitos do "Design Thinking”, que consiste em um conjunto de estágios (empatia, definição, ideação, prototipagem e teste) para solucionar situações problema (Brown, 2017). Na dinâmica, os alunos foram divididos em equipes de cinco integrantes e trabalhou-se com o tema "melhoria dos processos de recolha e reciclagem de resíduos sólidos". A dinâmica teve como objetivo imergir os participantes em uma estratégia reflexiva e colaborativa para resoluções que problemas, possibilitando o trabalho em equipe e soluções inovadoras (Oliveira, 2014; Sköldberg, Woodilla \& Çetinkaya, 2013; Brown \& Wyatt, 2010).

A realização da dinâmica ocorreu nas dependências da instituição de ensino em uma sala de "Co-working” com estrutura diferenciada e projetada para estimular o trabalho colaborativo e fomentar ideias. Os alunos foram divididos em cinco equipes com igual número de membros e ocuparam-se nas etapas do DT (empatia, definição, ideação, prototipagem e teste) para apresentar soluções a problemática “Como melhorar a coleta e reciclagem de resíduos sólidos urbanos?”. O tema em questão foi escolhido pois, além das questões sociais e sustentáveis, em 2020 evoca-se o vigésimo ano da instituição da Política Nacional de Resíduos Sólidos, Lei nº 12.305 de 2010.

Posteriormente à dinâmica, foi estabelecida uma sequência didática com cinco aulas contextualizadas (Tabela 1) com duas horas de duração onde discutiu-se a aplicação de conceitos voltados a metodologia de pesquisa em engenharia para: a) reconhecer necessidades, b) definição do problema de pesquisa; c) proposição de alternativas para solução do problema, d) implementação, e) testar e avaliar as alternativas; f) fechamento e conclusão. Estes aspectos foram explorados horizontalmente e relacionados verticalmente aos processos do PMBOK já elencados e as fases do ciclo de vida do projeto. 
Tabela 1: Temas apresentados na sequência didática para desenvolvimento dos projetos

\begin{tabular}{cl}
\hline Aula & \multicolumn{1}{c}{ Tema } \\
\hline 1 & Metodologia de pesquisa e desenvolvimento de projetos em engenharia \\
2 & Fase 1: Processos de iniciação de projetos \\
3 & Fase 2: Processos de organização e preparação de projetos \\
4 & Fase 3 e 4: Execução e encerramento de projetos \\
5 & Ferramentas gerenciais aplicadas à projetos \\
\hline
\end{tabular}

Fonte: Autores (2021).

Nas aulas foram abordados desde o desenvolvimento do termo de abertura do projeto, transpassando pelo gerenciamento de conhecimento, escopo, sequenciamento de atividades, qualidade, desenvolvimento da equipe, identificação de "stakeholders", etc. Além destas, foram demonstradas ferramentas como matriz SWOT e modelos de negócios Canvas. Ressalta-se que em todas foram incentivadas participações ativas dos alunos para promover seu envolvimento nos processos de ensino e aprendizagem (Barbosa \& Moura, 2013).

Nas etapas seguintes, as mesmas equipes voltaram-se para a escolha dos temas dos projetos para os TCCs, todos direcionados para a recuperação e revalorização de resíduos sólidos oriundos de diversos processos industriais. Neste momento iniciaram-se o desenvolvimento do termo de abertura do projeto, levantamento das atividades e prazos necessários para cumprimento dos objetivos e tarefas, organização das etapas experimentais e testes utilizando metodologia kanban e diagrama de Gantt (Peinado \& Graeml, 2007), análise SWOT e modelo de negócios (Hofrichter, 2017; Rosa, 2014). Além disto, houve o registro das atividades realizadas utilizando um "diário de bordo" estruturado para incluir os registros de reuniões, atividades experimentais, resultados, problemas e soluções (Duarte, 2018).

Ao final da $32^{\mathrm{a}}$ semana de trabalho foi aplicado um questionário para os alunos tratando-se de uma avaliação de desempenho dos membros das equipes ao longo do desenvolvimento dos projetos. Para tal, foram utilizados testes do tipo Likert em uma escala de 1 (discordo totalmente) a 5 (concordo totalmente) no qual o aluno se auto avaliava assim como avaliava os demais membros da equipe em dez "soft-skills" requeridas nos ambientes de trabalho (Robles, 2012): comunicação, cortesia, flexibilidade, integridade, habilidades interpessoais, atitude positiva, profissionalismo, responsabilidade, trabalho em equipe e ética. Também foram adicionadas duas categorias relacionadas com "hard skills": aptidão técnica e participação na elaboração de relatórios e diário de bordo. As respostas foram tabuladas utilizando suas médias, desvio padrão e coeficientes de variação $(\mathrm{CV} \%)$ e estas foram utilizadas para fazer uma análise hierárquica de agrupamentos (HCA) para reconhecimento de padrões utilizando o software Minitab versão 18.

\section{Resultados e Discussão}

O mercado de trabalho cada vez mais necessita de capacitação daqueles que participarão no desenvolvimento de projetos e nesta perspectiva a formação de recursos humanos para atuar nestes pode ter origem nos cursos da voltados a Educação Profissional e Tecnológica (EPT) (Grabowski, 2014).

No Brasil, o artigo nº 39 da Lei no 9394 de 1998 (Lei de Diretrizes e Bases da Educação Nacional) conceitua essa como uma modalidade de ensino que abrange diferentes níveis de ensino (i.e. formação inicial e continuada, cursos técnicos de ensino médio, cursos de graduação e pós-graduação) estabelecidos em eixos de formação com enfoque no trabalho e emprego, ciência e tecnologia e que teoria e prática devem ser inerentes uma à outra. Neste sentido, a composição curricular dos cursos da EPT deve ser estruturada para atender estes princípios sendo que as instituições de ensino possuem autonomia na concepção desta composição e requerer, por exemplo, o trabalho de conclusão de curso como um dos requisitos para diplomação (Pacheco, 2012). 
Habitualmente, os TCCs são interpretados pelos alunos como algo apenas protocolar como um dos requisitos para a finalização do curso, onde na maioria dos casos é exigida a aplicação de componentes curriculares ${ }^{1}$. Isto se deve, em parte, pois no início do desenvolvimento dos mesmos constantemente se repetem a transmissão de orientações sobre os modelos requeridos, uma introdução a metodologia de pesquisa, o número de horas que serão dedicadas ao trabalho, sua execução propriamente dita e a sua apresentação. Deste modo, perde-se a oportunidade de conciliar o que foi diretamente estudado com o desenvolvimento indireto de habilidades profissionais uma vez que não são inseridas metodologias que contribuam para isso e, então, diminuindo o engajamento dos alunos que não vislumbram o TCC como algo enriquecedor para seu exercício profissional.

Estas premissas serviram como base para este trabalho e geraram uma sequência de ações para contribuir na formação de profissionais (não mais alunos) cientes de que existem estratégias gerenciais, iniciando-se como uma maneira não convencional de iniciar os projetos da EPT por meio da utilização do "Design Thinking”, seguido da implementação das etapas e ferramentas para planejamento, execução e controle dos TCCs.

\section{1 “Design Thinkng” como dinâmica motivadora inicial}

As demandas por novas estratégias de trabalho aumentam a medida que o desenvolvimento de projetos torna-se cada vez mais complexo (Lukosevicius, Soares, Joia, 2018), sendo que esta complexidade é resultado da interdependência de áreas, da volatilidade e imprevisibilidade das situações assim como a ambiguidade que as diferencia sutilmente não possibilitando somente responde-las positiva ou negativamente. Tais conceitos estão interligados ao paradigma do mundo VUCA ${ }^{2}$ que requer transformações na cultura organizacional (Llop, et al., 2017).

Neste sentido, o "Design Thinking" [DT] destaca-se como estratégia centrada no humano, integrada e colaborativa. Além disto, o DT na educação profissional e tecnológica pode exercitar a empatia ao focar no usuário interessado na resolução de uma situação problema tornando-o coparticipante do processo (Martins, 2016; Oliveira, 2014; Dym, et al., 2005). Estas foram as principais intenções da utilização do DT como dinâmica inicial, pois permitiu envolver os alunos da concepção, experimentação e demonstração de ideias para a problemática apresentada cujo tema enquadra-se na necessidade de integração da inovação e do desenvolvimento sustentável nas cadeias produtivas das indústrias químicas (Dias, Guimarães e Santos, 2012; Menezes, et al., 2011).

A primeira etapa (empatia) consistiu em apresentar o desafio e instigar como poderiam abordá-lo do ponto de vista de outras pessoas, propondo-se que entrevistassem membros de outras equipes para entender quais eram as necessidades e chegar aos "insights" que direcionariam para soluções. Nesta etapa, a intenção era elucidar quais eram os principais problemas das pessoas em relação ao direcionamento dos resíduos sólidos.

Na segunda etapa houve a interpretação e definição de significados, oportunidades e desafios para, então, seguir à etapa de ideação onde utilizou-se uma sessão de "brainstorming" para definir em conjunto a estratégia de solução. Para tal, após a geração das ideias empregou-se uma matriz de priorização (Brown, 2017) para determinar quais eram mais empáticas e inovadoras, conforme esquematizado na Figura 1.

\footnotetext{
${ }^{1}$ Opinião pessoal do autor baseado na sua experiência como docente e orientador de trabalhos de conclusão de curso.

${ }^{2}$ Sigla derivada dos termos em língua inglesa "Volatility, Uncertainty, Complexity e Ambiguity".
} 
Figura 1: Matriz de priorização para separar as ideias entre níveis mais (+) e menos (-) em relação aos quesitos inovação e empatia.

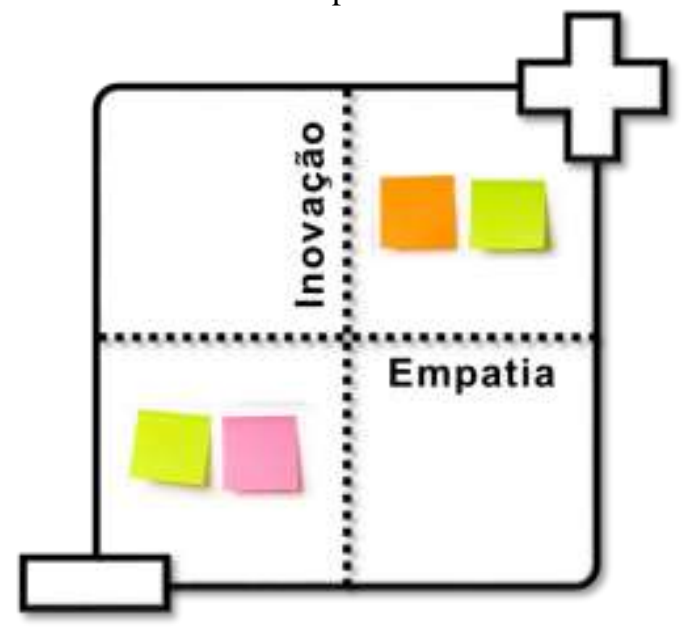

Fonte: Baseado em Brown (2017).

Posteriormente houve a prototipagem das soluções com maior inovação e que atendesse a demanda dos entrevistados inicialmente, ou seja que apresentasse maior empatia às necessidades observadas, seguido da etapa de testes e demonstração onde discutiu-se coletivamente (“feedback" colaborativo) os pontos fortes e fracos assim como dúvidas e ideias para as soluções apresentadas.

A importância da dinâmica nesta fase do TCC consistiu em apresentar uma sequência de atividades relacionadas a um objetivo, similarmente ao aplicado em projetos seguindo as orientações do PMBOK, por exemplo. Além disso, a dinâmica trabalhou com situações para atividades individuais e em equipe direcionadas para pensamentos divergentes e convergentes, ou seja, atividades onde não há um consenso (i.e. processos de opinião pessoal) e outras em que este deverá ocorrer (i.e. processos colaborativos), respectivamente (Figura 2).

Figura 2: Esquematização das atividades de caráter individual e em equipe e sua relação com os tipos de pensamento.

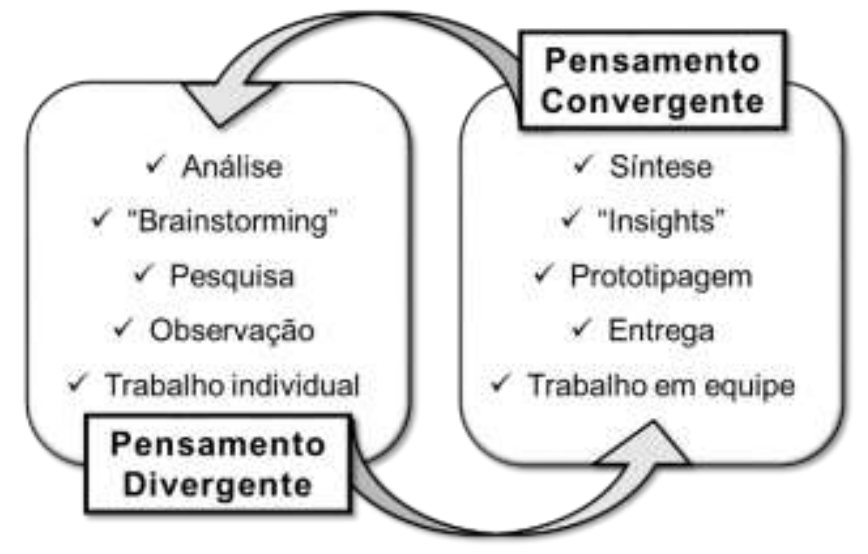

Fonte: Baseado em Oliveira (2014).

O "feedback" dos alunos foi bastante positivo uma vez que percebeu-se que os conceitos e atividades aplicadas são fundamentais para o desenvolvimento de projetos, evidenciando que a pluralidade de observações e análises pessoais coparticipam da elaboração de soluções às demandas iniciais (Oliveira, 2014; Sköldberg, et al., 2013; Dym, et al., 2005). 


\subsection{Sequência didática sobre o desenvolvimento de projetos}

Após a imersão utilizando o DT prosseguiu-se com as atividades da disciplina utilizando a sequência didática mencionada na metodologia para configurar o TCC como elemento (real) de aprendizado. Optou-se por esta estratégia pois a dinâmica anterior possibilitou contextualizar as dificuldades e oportunidades aos conceitos para o desenvolvimento de projetos.

Como já descrito na seção anterior, este trabalho explorou horizontalmente etapas da metodologia de pesquisa em engenharia e verticalmente as fases do ciclo de vida de projetos indicadas pelo PMBOK, complementando-se umas às outras. A Figura 3 esquematiza esta estratégia demonstrando também as saídas do gerenciamento em função do tempo de desenvolvimento do projeto.

Figura 3: Integração entre as fases do ciclo de vida de projetos e metodologia de pesquisa em engenharia.

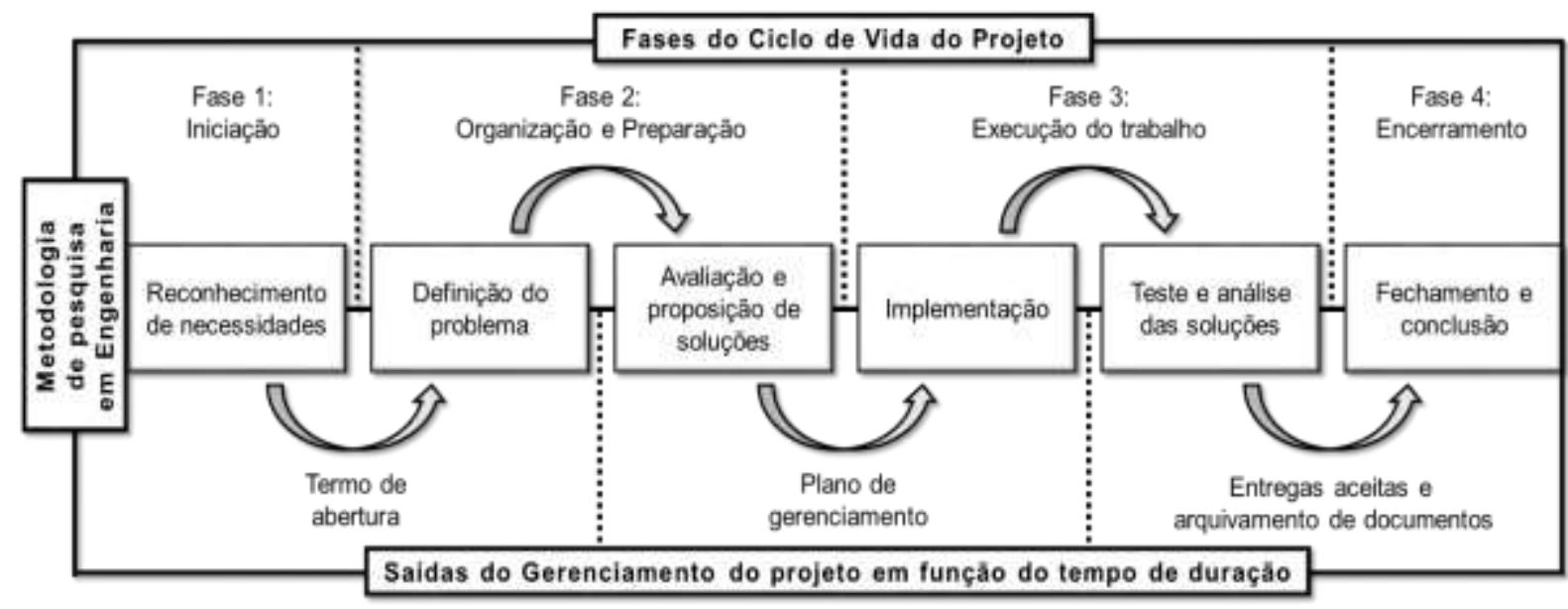

Fonte: Autores (2021).

A organização conceitual demonstrada na Figura 4 serviu como base para as aulas da sequência didática inicial. Na mesma foram expostos os conceitos voltados a metodologia de pesquisa em engenharia em uma perspectiva ativa, procurando questionar as etapas. Deste modo o reconhecimento de necessidades, por exemplo, pautou-se na observação de possíveis melhorias para demandas locais. Definidas as situações problema, os temas foram especificados e então propostas soluções baseado em pesquisas de outros trabalhos e avaliados pontos fortes e fracos e quais seriam os impactos e dificuldades de implementação destas alternativas.

Nas aulas seguintes, sob a ótica da gestão de projetos e direcionamento do PMBOK, tais aspectos foram relacionados às fases do ciclo de vida de projetos (iniciação, organização, execução e encerramento). Ficou evidente que ambos os conteúdos (metodologia de projetos em engenharia e os conceitos de gestão do PMBOK) são uníssonos ao ter como objetivo a identificação e resolução de uma demanda por produto ou serviço, diferindo-se nas estratégias gerenciais utilizadas. Se por um lado o método de engenharia, por exemplo, ocupa-se de organizar as melhores condições para um experimento e a demonstração técnica desta, as estratégias do PMBOK orientam quanto a interação entre as etapas do projeto, análise de risco, gestão do tempo, entrega de documentos e integração a outras esferas como por exemplo a administração e modelo de negócios.

Esta relação foi contextualizada a partir de estudos de caso e demonstração da documentação necessária, bem como motivando os alunos a trazerem exemplos profissionais e que, de fato, estes conteúdos são aplicáveis e necessários. Partiu-se do princípio que a contextualização e utilização de situações problema reforça a aprendizagem significativa e contrapõem-se ao ensino unicamente baseado nas "hard skills" pelo fato de possibilitar o exercício de habilidades para resolver problemas e conduzir projetos ativamente (Barbosa \& Moura, 2013). 


\subsection{Iniciação, organização e preparação dos projetos de TCC}

Posteriormente a dinâmica inicial utilizando o DT onde houve uma imersão no desenvolvimento de projetos, bem como da sequência de aulas contextualizadas sobre as etapas da metodologia de projetos e relacionadas ao PMBOK iniciaram-se as atividades de iniciação dos projetos de TCCs utilizando os conceitos teóricos e conhecimentos práticos.

Para tal, houve a apresentação do período em que os projetos deveriam ser desenvolvidos e as atividades e tarefas para serem desempenhadas e entregues, respectivamente. Neste momento houve uma reunião para estabelecer os prazos para essas e conjuntamente foi elaborado um diagrama de Gantt, uma ferramenta bastante útil pois demonstra visualmente as atividades e sequencia de trabalho (Peinado \& Graeml, 2007). A Figura 4 ilustra o diagrama de Gantt elaborado utilizando um "template" do software Excel $^{3}$.

Figura 4: Diagrama de Gantt com as atividades dos TCCs elaborado com a participação dos alunos.

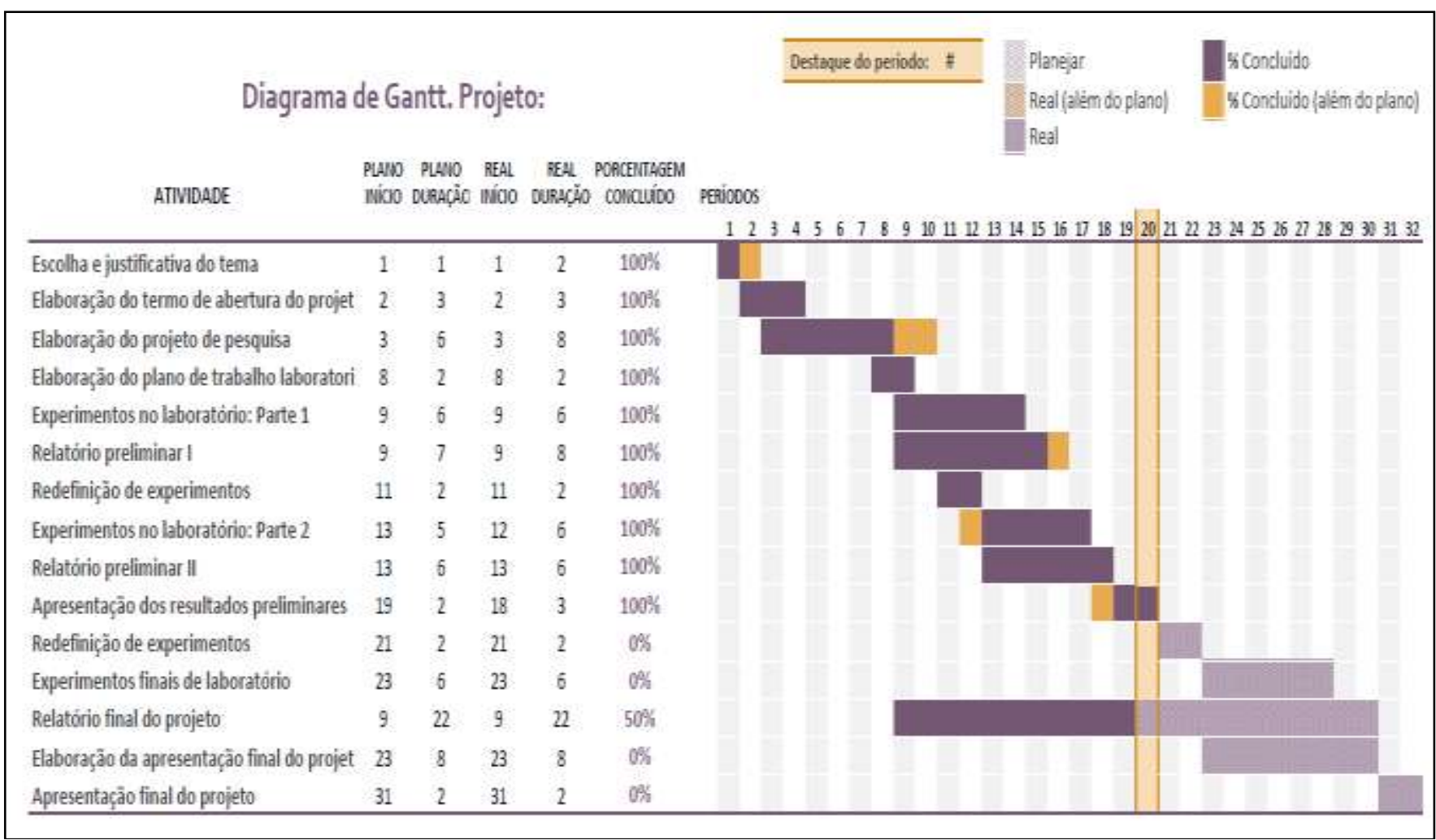

Fonte: Autores (2021).

No diagrama de Gantt (Figura 4) foi possível indicar horizontalmente os períodos planejados, reais de execução e além do prazo e as porcentagens de conclusão reais e além do prazo estabelecido durante as 32 semanas de duração dos projetos. Também foi possível indicar as atividades e entregas que seriam realizadas e em qual período de tempo a começar pela escolha e justificativa do tema ( $1^{\mathrm{a}}$ semana) do TCCs (abordando os resíduos sólidos) onde cada equipe se concentrou em um material (gesso, plásticos, borracha, tintas, tecidos). Posteriormente houve a elaboração do projeto de pesquisa da $3^{\mathrm{a}}$ a $6^{\mathrm{a}}$ semana de trabalho (estendida para a $8^{\mathrm{a}}$ semana, neste caso) e planejamento do plano de trabalho no laboratório. Destaca-se, também, os períodos das entregas dos resultados preliminares e relatório final dos TCCs.

${ }^{3}$ Planejador de projetos de Gantt. Disponível em:< https://templates.office.com/pt-br/planejador-de-projetos-de-gantt-tm02887601>. 
Definidos os temas e organizadas as atividades e entregas, deu-se a elaboração do Termo de abertura do Projeto utilizando um modelo elaborado internamente, conforme ilustrado na Figura 5.

Figura 5: Modelo do Termo de Abertura do Projeto utilizado para os projetos de TCC.

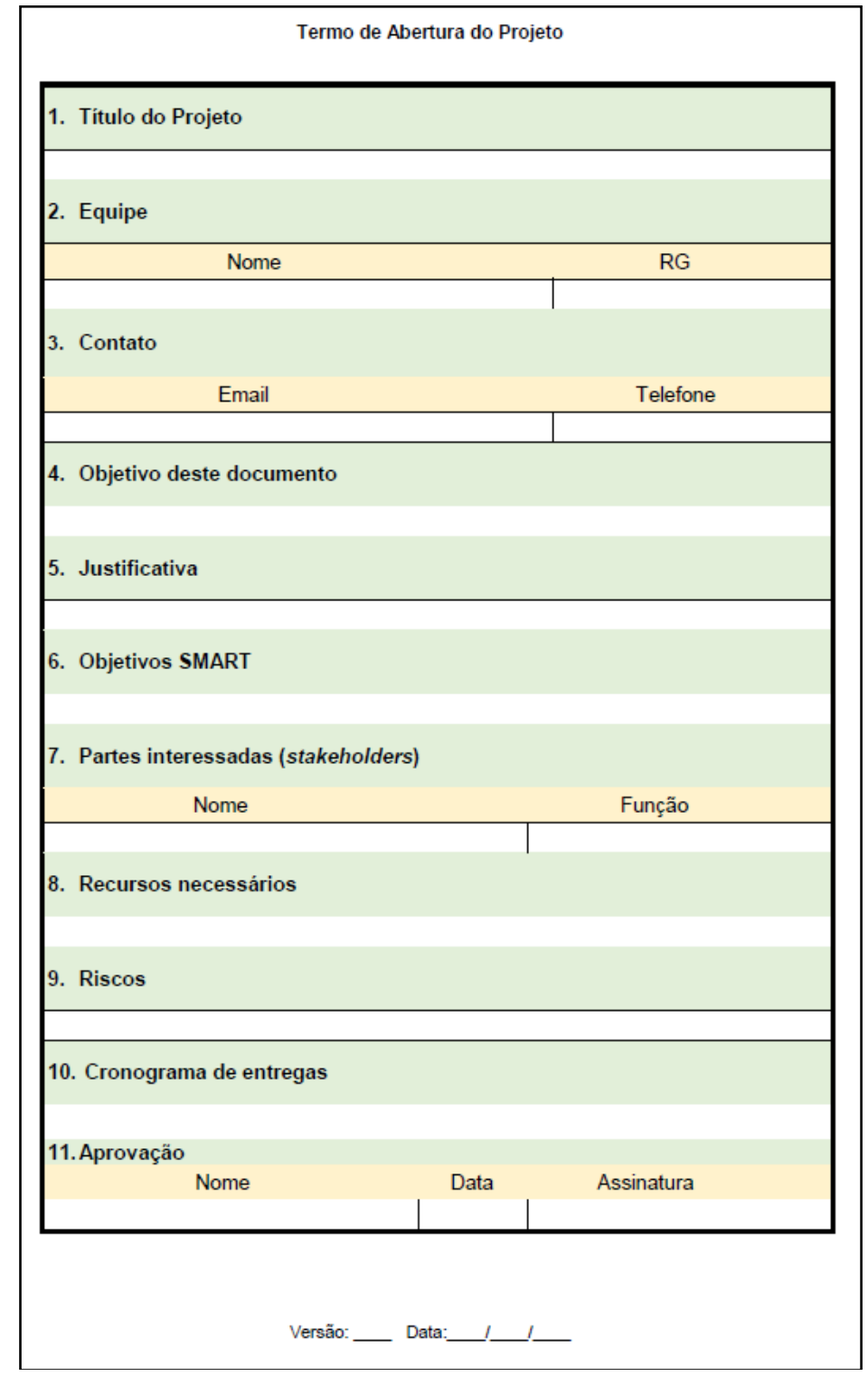

Fonte: Autores (2021).

O formalismo do Termo de Abertura do Projeto denota profissionalismo, fato observado pelos alunos no seu preenchimento que necessitava de suas informações de identificação e contato, o objetivo do documento (atribuição dos responsáveis e premissas principais), da justificativa (situação problema que o projeto visa resolver) e objetivos SMART ("Specific", "Measurable", "Assignable", "Realistic" e "Time-related", no caso relacionando as especificidades, metas, por quem e quando seriam desenvolvidas as atividades).

Na Figura 5 também identifica-se a necessidade de indicar os "stakeholders", ou seja, as partes interessadas do projeto. Esta definição é importante pois configura-se como um dos quesitos de maior complexidade no desenvolvimento de projetos (Lukosevicius, Soares \& Joia, 2018). No caso dos TCCs elaborados na instituição de ensino, esses foram delimitados entre 
professores, coordenação de curso, órgão ambientais locais e industrias que originam os resíduos utilizados. Também foram inseridos campos para os recursos necessários (equipamentos de laboratório, infraestrutura para testes, etc) e quais seriam os riscos (ameaças e oportunidades) do projeto, encerrando-se com o cronograma de entregas baseado na distribuição temporal do Diagrama de Gantt.

Paralelamente a elaboração do projeto de pesquisa, foi solicitado que desenvolvessem uma análise de risco baseada na matriz SWOT pois trata-se de uma importante ferramenta para o planejamento estratégico ao considerar o ambiente interno com suas forças e fraquezas e o ambiente externo com suas oportunidades e ameaças no desenvolvimento dos projetos (Silva Filho, 2015; Rosa, 2014; Fernandes, et al., 2013).

Quanto a análise do ambiente interno, foram apontados diversos fatores como determinantes para a execução do projeto, conforme síntese apresentada na Tabela 2. No geral as forças resumem-se em aspectos técnicos e experiências de trabalhos anteriores, já as fraquezas indicam as dificuldades para testes, tempo e acesso a espaços e informações sobre os temas, assim como o possível cansaço para execução dos mesmos pois as equipes na grande maioria são compostas por alunos que trabalham durante o dia e cursam a faculdade no período noturno.

Tabela 2: Síntese da análise para ambiente interno com as forças e fraquezas da análise SWOT.

\begin{tabular}{lll}
\hline \multicolumn{1}{c}{ Forças } & \multicolumn{1}{c}{ Fraquezas } \\
\hline$\checkmark$ Equipe técnica estruturada & $\checkmark$ & Estrutura para alguns testes \\
$\checkmark$ "Know-how" no tratamento de resíduos & $\checkmark$ & Dependência de requisições \\
$\checkmark$ Capacidade de aprendizado & $\checkmark$ & Falta de estudos na área \\
$\checkmark$ Fácil acesso a insumos & $\checkmark$ & Tempo hábil para o projeto \\
$\checkmark$ Comunicação & $\checkmark$ Organização \\
$\checkmark$ Tomada de decisão & $\checkmark$ Cansaço \\
$\checkmark$ Vivência industrial & $\checkmark$ Recursos \\
\hline
\end{tabular}

Fonte: Autores (2021).

$\mathrm{Na}$ análise do ambiente externo (Tabela 3) foram apontadas como principais oportunidades a possibilidade de colaborações com outras instituições e aprendizado prático bem como o aumento do pensamento sustentável em relação a revalorização dos resíduos, porém foram apontadas como ameaças essencialmente a falta de estudos na área, atendimento a legislação ambiental e a aceitação de produtos oriundos de resíduos.

Tabela 3: Síntese da análise para ambiente externo com as oportunidades e ameaças da análise SWOT.

\begin{tabular}{cllll}
\hline \multicolumn{3}{c}{ Oportunidades } & & \multicolumn{1}{c}{ Ameaças } \\
\hline$\checkmark$ & Rede de colaborações (prefeitura, outros & $\checkmark$ & Ineficiência da separação dos resíduos \\
& laboratórios/grupos de pesquisa, órgãos ambientais, & $\checkmark$ & Falta de estudos sobre o resíduo \\
& associações, etc) & $\checkmark$ & Atendimento a legislação \\
$\checkmark$ & Aumento do pensamento sustentável & $\checkmark$ & Agregação de valor ao resíduo \\
$\checkmark$ & Produtos inovadores & $\checkmark$ & Aceitação da solução tecnológica \\
$\checkmark$ & Atendimento a legislação ambiental & $\checkmark$ & Pouca literatura disponível \\
$\checkmark$ & Redução de impactos ambientais & $\checkmark$ & Tempo hábil para execução \\
$\checkmark$ & Aprendizado & $\checkmark$ & \\
$\checkmark$ Estreitamento entre teoria e prática & & & \\
\hline
\end{tabular}

Fonte: Autores (2021).

Além da análise SWOT, foi desenvolvido o modelo de negócio utilizando a abordagem Canvas para gestão de projetos e indicar os requisitos e relação dos temas com seus "stakeholders" de forma gráfica e rápida (Moraes, et al., 2017; Rosa, 2014). 
Para tal, utilizou-se o guia de modelo de negócios do Serviço Brasileiro de Apoio às Micro e Pequenas Empresas [SEBRAE] (Rosa, 2014). A Figura 6 ilustra genericamente os planos de negócios dos temas de pesquisa sobre os resíduos, onde visualizamse as relações dos projetos com suas possíveis parcerias, atividades-chave, recursos, proposição de valor, relacionamento com clientes custos e receitas.

Figura 6: Representação do modelo de negócios Canvas dos projetos de TCC.

\begin{tabular}{|c|c|c|c|c|}
\hline \multirow{2}{*}{$\begin{array}{c}\text { Parcerias } \\
\text { Principais } \\
\text { Empresas } \\
\text { fornecedoras de } \\
\text { resíduos. } \\
\text { Terceirização } \\
\text { de serviços. } \\
\text { Indústrias que } \\
\text { geram resíduos. } \\
\text { Fornecedores } \\
\text { de reagentes } \\
\text { essenciais para } \\
\text { o processo. }\end{array}$} & $\begin{array}{l}\text { Atividades-Chave } \\
\text { Produção de novos } \\
\text { materiais; } \\
\text { Redução de } \\
\text { resíduos. }\end{array}$ & \multirow{2}{*}{$\begin{array}{l}\text { Revalorização } \\
\text { dos resíduos; } \\
\text { Sustentabilidade; } \\
\text { Redução de } \\
\text { resíduos em } \\
\text { aterros. }\end{array}$} & $\begin{array}{l}\text { Relacionamento } \\
\text { Com Clientes } \\
\\
\text { Segmento } \\
\text { diversificado de } \\
\text { diversas áreas da } \\
\text { indústria e } \\
\text { sociedade. }\end{array}$ & \multirow[t]{2}{*}{$\begin{array}{l}\text { Segmento de } \\
\text { Clientes } \\
\text { Empresas que } \\
\text { buscam uma } \\
\text { forma mais } \\
\text { sustentável } \\
\text { proveniente de } \\
\text { resíduos. }\end{array}$} \\
\hline & $\begin{array}{c}\text { Recursos } \\
\text { Principais } \\
\text { Resíduos de tecido } \\
\text { de algodão; } \\
\text { Recursos humanos; } \\
\text { Equipamentos. }\end{array}$ & & $\begin{array}{c}\text { Canais } \\
\text { Distribuidores e } \\
\text { empresas que } \\
\text { comercializam o } \\
\text { produto final. }\end{array}$ & \\
\hline \multicolumn{2}{|c|}{$\begin{array}{c}\text { Estrutura de Custos } \\
\text { Infraestrutura; Reagentes e } \\
\text { equipamentos; Obtenção dos resíduos }\end{array}$} & \multicolumn{3}{|c|}{$\begin{array}{l}\text { Fontes de Receita } \\
\begin{array}{c}\text { Vendas diretas de dos novos materiais para empresas e } \\
\text { consumidores em geral; }\end{array}\end{array}$} \\
\hline
\end{tabular}

Fonte: Autores (2021).

De maneira geral, as estratégias gerenciais utilizadas nas etapas de início e preparação dos projetos se prestaram a inserir formas de planejamento estratégico e inovação na maneira de conduzir trabalhos na EPT. As observações levantadas na matriz SWOT e do modelo de negócios foram incorporadas aos projetos de pesquisa e embasaram discussões além das puramente acadêmicas, propiciando a compreensão e avaliação crítica do projeto como algo pertencente há contextos além dos aplicados habitualmente nos TCCs como as perspectivas sociais e econômicas.

\subsection{Execução, controle e encerramento dos TCCs}

As etapas de desenvolvimento práticas do projeto ocorreram após sua preparação conforme organização demonstrada no diagrama de Gantt (Figura 4). Iniciaram-se os experimentos no laboratório e a elaboração dos documentos de registro de atividades, como o diário de bordo. Ressalta-se que durante toda esta fase houveram reuniões semanais sobre o andamento dos projetos durante as aulas, algo que imputou a necessidade de organização e seguimento do planejamento realizado.

Para controle das atividades, utilizou-se a metodologia Kanban que consiste em indicar de maneira simplificada as atividades que estão à espera de realização, em andamento e as que foram concluídas, sequencialmente (Peinado \& Graeml, 2007). A Figura 7 demonstra a utilização da metodologia através do software Trello, onde é possível observar as atividades e seu status em formato de cartões que são deslocados (puxados) de uma categoria para outra conforme avança seu desenvolvimento. A estratégia demonstrou-se de grande utilidade ao propiciar um controle visual das atividades e também por indicar os responsáveis pela sua execução. 
Figura 7: Representação dos seguimentos da metodologia kanban utilizada no desenvolvimento dos TCCs.

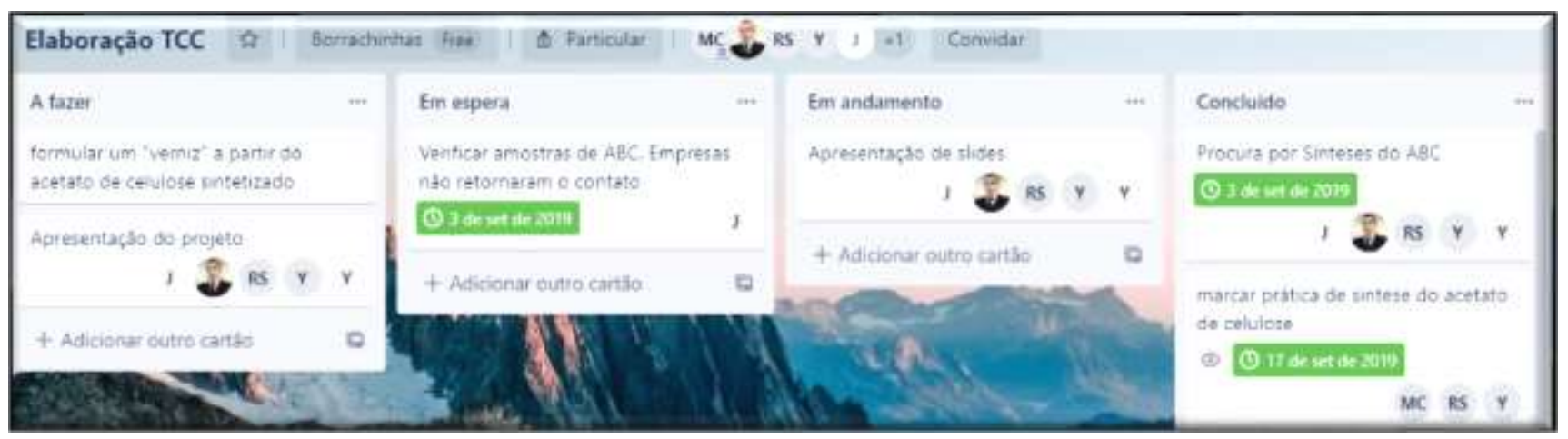

Fonte: Autores (2021).

Ao final da $18^{\mathrm{a}}$ semana de execução dos projetos foi aplicado um questionário para evidenciar o conhecimento das equipes em relação aos projetos. Primeiramente, um conjunto de questões específicas como saber o título do projeto, qual sua justificativa e quais as etapas e experimentos já executados e futuras foram inseridas para comparar as informações entre os membros das equipes e indicar se haveria sintonia daquilo que foi planejado e executado. Foi observado que as respostas divergiram bastante levando-os a perceber que apesar de estarem no mesmo projeto haveria a necessidade de maior alinhamento das ações e ciência daquilo que que já havia realizado com as demais etapas a realizar.

No mesmo questionário os alunos também foram indagados sobre as dificuldades enfrentadas na execução do projeto, como este se relaciona com outras disciplinas do curso, qual a importância do TCC na formação, ou seja, questões que abrangiam a formação geral do aluno e não restrita somente a execução técnica do projeto. Notou-se que houve facilidade de relaciona-lo a outras disciplinas pois a contextualização sempre foi uma das premissas para o desenvolvimento dos projetos relacionando a teoria aos aspectos práticos. Quanto as dificuldades apontadas, muitas se relacionaram com as fraquezas e ameaças colocadas na matriz SWOT, demonstrando que as etapas de planejamento são, de fato, importantes ao prever tais situações. Ainda nesta seção do questionário, a importância dos TCCs na forma em que estava sendo desenvolvido foi colocada como algo que representa um trabalho em equipe nas empresas onde o projeto deve ser estudado, testado e concluído em conjunto. Além disso, as respostas também evidenciaram que o TCC permite ampliar a visão referente a trabalho em equipe, organização, prazos, lidar com sucessos e frustrações, competências essenciais no mercado de trabalho.

Simultaneamente às atividades de laboratório e apresentações de resultados prévios, na $8^{\mathrm{a}}$ semana de trabalho iniciouse a elaboração do relatório final dos TCCs utilizando a plataforma Google Docs. A estratégia mostrou-se vantajosa pois permitiu a edição online e colaborativa do documento sendo possível verificar as contribuições individuais e o avanço na elaboração do mesmo, sendo este adicionado no diagrama de Gantt (Figura 4). Além disto, esta estratégia forneceu "feedback" bastante positivo pelo dinamismo e de não necessitar de revisões individuais segmentadas já que a edição em tempo real era efetuada simultaneamente por toda a equipe propiciando a discussão coletiva dos resultados, atribuição de causas e efeitos nos experimentos e formatação segundo as normas estabelecidas.

\subsection{Avaliação das competências comportamentais - Soft skills}

Ao final da $32^{\mathrm{a}}$ semana de trabalho, realizou-se com os alunos um questionário para fornecer "feedback" entre aquilo que o aluno julga e aquilo que julgam do mesmo (Leme, 2006) buscando avaliar competências profissionais atreladas tanto a "soft-skills" quanto "hard-skills". O questionário foi organizado para que o aluno atribuísse notas variando de 1 a 5 , ou seja 
desde um nível onde o quesito estaria aquém da expectativa passando por níveis intermediários (regulares) até o nível “outstanding”. As respostas foram compiladas e realizados os cálculos estatísticos cujos resultados são listados na Tabela 4.

Tabela 4: Dados estatísticos da avaliação de competências das equipes.

\begin{tabular}{lccc}
\hline "Soft skill" & Média & Desvio padrão & CV\% \\
\hline Aptidão técnica & 3,99 & 0,71 & 17,71 \\
Atitude positiva & 3,49 & 0,87 & 24,90 \\
Comunicação & 3,83 & 0,87 & 22,73 \\
Cortesia & 4,03 & 0,81 & 20,20 \\
Ética & 4,14 & 0,82 & 19,82 \\
Flexibilidade & 4,34 & 0,80 & 18,54 \\
Habilidades interpessoais & 3,69 & 0,77 & 20,74 \\
Integridade & 4,27 & 0,64 & 14,99 \\
Profissionalismo & 3,99 & 0,77 & 19,19 \\
Relatórios e diário de bordo & 4,17 & 0,54 & 13,06 \\
Responsabilidade & 4,02 & 0,85 & 21,17 \\
Trabalho em equipe & 3,72 & 0,93 & 25,00 \\
\hline
\end{tabular}

Nota: $\mathrm{CV} \%$ = coeficiente de variação. Fonte: Autores (2021).

Apesar de serem consideradas intangíveis (Robles, 2012) as competências profissionais listadas na Tabela 4 foram avaliadas e os dados demonstraram que as médias variaram de 3,49 a 4,34 com variações na faixa $13,06 \%$ a $25,00 \%$. Considerando os desvios houveram alunos que obtiveram notas médias próximas a 3, ou seja, considerados regulares por si e pelos demais integrantes da equipe e necessitam de maior atenção para desenvolver estas competências em comparação aos que obtiveram médias próximas a 5 algo que pode ser realizado em conjunto com os outros integrantes da equipe em conjunto com os professores e corpo técnico da instituição de ensino (Bancino \& Zevalkink, 2007).

Positivamente, obtiveram-se médias altas na ética, flexibilidade e integridade indicando que a maioria dos alunos apresenta características ligadas a adaptabilidade e valores pessoais sendo "soft-skills" essenciais no ambiente de trabalho (Ritter et. al., 2018; Robles, 2012; Laker \& Powell, 2011). Contrariamente, médias baixas nos quesitos atitudes positivas, habilidades interpessoais e trabalho em equipe denotam que o trabalho coletivo ainda é um desafio às individualidades que se sobrepõem aos aspectos cooperativos e de pensamento convergente necessários ao desenvolvimento dos projetos.

Por fim, relacionaram-se todos os resultados em uma análise hierárquica de agrupamentos com o objetivo de demonstrar padrões entre as médias da avaliação de competências comportamentais. O resultado da análise é apresentado no dendograma (Figura 8) que demonstra a similaridade dos quesitos avaliados. 
Figura 8: Similaridade dos quesitos utilizados para avaliação das competências comportamentais.

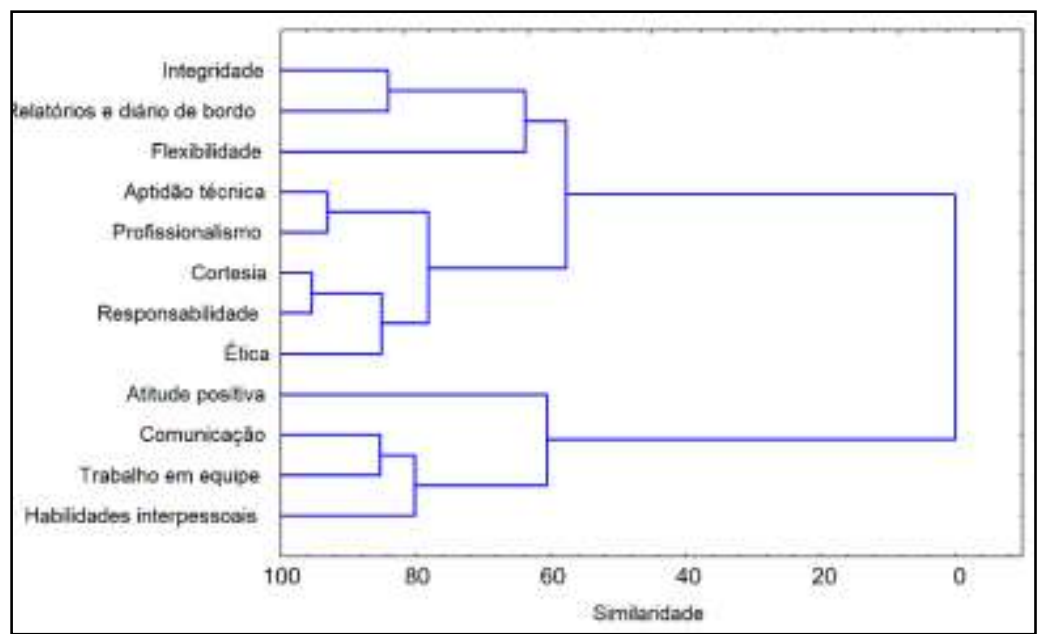

Fonte: Autores (2021).

As informações contidas na Figura 8 indicam três grandes agrupamentos. O primeiro é composto pelos quesitos integridade e participação nos relatórios e diário de bordo ( $\sim 80 \%$ de similaridade) e flexibilidade ( $\sim 60 \%$ de similaridade com os anteriores), indicando que o registro das atividades necessita ser correta e ao mesmo tempo há a necessidade de se adaptar às mudanças no desenvolvimento dos projetos.

Um segundo agrupamento é composto pelos quesitos aptidão técnica e profissionalismo, cortesia e responsabilidade ( $~ 90 \%$ de similaridade) juntamente com a ética profissional (> 80\% de similaridade com as demais). Estes resultados sinalizam que os aspectos técnicos ("hard skills") são diretamente atrelados ao saber fazer eticamente responsável e equilibrado em relação ao outro e ao projeto.

Por último encontra-se um agrupamento com $0 \%$ de similaridade com os anteriores onde foram agrupadas a comunicação, trabalho em equipe e habilidades interpessoais ( $80 \%$ de similaridade) juntamente com a atitude positiva (60\% de similaridade com as demais). Tais resultados expressam a necessidade de acompanhamento e treinamento dos alunos pois reforçam o desafio das características individuais influenciarem no resultado dos projetos. As atitudes positivas relacionam-se aos de motivação ao trabalho e alinham-se as habilidades interpessoais na resolução de conflitos na equipe do projeto, por exemplo e tais aspectos estão diretamente relacionados à necessidade de comunicação clara e concisa para um trabalho efetivamente cooperativo em equipe.

A estratégia de avaliação utilizada mostrou-se positiva ao demonstrar aos alunos os aspectos aqui levantados e discutidos, sendo possível aos mesmos reconhecer-se nos agrupamentos bem como debater formas de aprimoramento humano e profissional.

\section{Considerações Finais}

Este trabalho pautou-se em desenvolver os projetos de conclusão de curso utilizando estratégias gerenciais do PMBOK e avaliar competências necessárias ao mercado de trabalho. Estas estratégias demonstraram-se benéficas no desenvolvimento dos TCCs ao incorporar ferramentas atreladas ao planejamento, análise de risco e de mercado, execução e controle dos projetos. De fato, a metodologia proposta pode ser utilizada em trabalhos futuros adequando-se as ferramentas gerenciais ao contexto educacional e às turmas envolvidas em trabalhos de conclusão de curso. Além disto, indiretamente foram contextualizadas e discutidas competências profissionais essenciais e que possibilitaram um currículo paralelo direcionado a teoria e prática profissional. Deste modo, este trabalho contribui para a concepção de uma educação profissional e tecnológica que supera o 
conceito de formação exclusivamente técnica para ser, de fato, integrada às necessidades do processo produtivo, sociais e humanas.

\section{Referências}

Almeida, L. S. (2002). Facilitar a aprendizagem: ajudar aos alunos a aprender e a pensar. Psicologia Escolar e Educacional, 6 (2), 155-165. https://doi.org/10.1590/S1413-85572002000200006

Andrews, J. \& Higson, H. (201)0. Graduate employability, Soft Skills versus Hard business knowledge: a european study. Higher Education in Europe, 33 (4), 411-422. https://doi.org/10.1080/03797720802522627

Bancino, R. \& Zevalkink, C. (2007). Soft Skills: The new curriculum for hard-core technical professionals. Techniques: Connecting Education and Careers, 82 (5), 20-22.

Barbosa, E.F. \& Moura, D.G. (2013). Metodologias ativas de aprendizagem na educação profissional e tecnológica. Boletim Técnico Senac, 39 (2), $48-67$.

Brown, T. (2017). Design Thinking: uma metodologia poderosa para decretar o fim das velhas ideias. Rio de Janeiro, Brasil: Alta Books.

Brown, T. \& Wyatt, J. (2010). Design Thinking for social innovation. https://ssir.org/articles/entry/design_thinking_for_social_innovation.

DeLuiz, N. (2001). O modelo de competências profissionais no mundo do trabalho e na educação: implicações para o currículo. Boletim Técnico do Senac, 27(3), 1-16.

Dias, S. L. F. G.; Guimarães, F. F. \& Santos, M. C. L. (2012). Inovação no desenvolvimento de produtos verdes: integrando competências ao longo da cadeia produtiva. Revista de Administração e Inovação, 9 (3), 129-153. https://www.revistas.usp.br/rai/article/view/79279

Duarte, E. A. (2018). Proposta de metodologia para o desenvolvimento de projetos de engenharia direcionada ao ensino médio e superior. Tese de doutorado em Engenharia Mecânica, Faculdade de Engenharia Mecânica, Universidade Estadual de Campinas, Campinas, SP, Brasil.

Dym, C. L.; Agogino, A. M.; Ozgur, E. \& Frey, D. D. (2005). Engineering design thinking, teaching, and learning. Journal of Engineering Education, 94 (1), 103-120. https://doi.org/10.1002/j.2168-9830.2005.tb00832.x

Fernandes, I. G. M.; Figueiredo, H. M.; Costa Jr, H. L.; Sanches, S.G. \& Brasil, A. (2013). Planejamento estratégico: análise SWOT. Revista Conexão Eletrônica, $10(1), 1464-1473$.

Gerhardt, E. \& Silveira, D. T. (2009). Métodos de pesquisa. Porto Alegre, Brasil: Editora da UFRGS.

Grabowski, G. (2014). Gestão e planejamento da educação profissional e tecnológica. Curitiba, Brasil: Instituto Federal do Paraná.

Hofrichter, M. (2017). Análise SWOT: quando usar e como fazer. Porto Alegre, Brasil: Editora Simplissimo.

Laker, D. R. \& Powell, J.L. (2011). The differences between hard and soft skills and their relative impact on training transfer. Human Resource Development Quarterly, 22 (1), 111-122. https://doi.org/10.1002/hrdq.20063

Leme, R. (2006). Avaliação de Desempenho com Foco em Competência. Rio de Janeiro, Brasil: Qualitymark.

Llop, R.; Grasas, Á.; Paassen, J.; Rosés, L. \& Araújo, M. S. 2017. O papel dos gestores atuais para sobreviver num ambiente VUCA. Revista de Ciências Empresariais e Jurídicas, 29, 7-33. https://doi.org/10.26537/rebules.vi29.2900

Lukosevicius, A. P.; Soares, C. P. \& Joia, L. A. (2018). Caracterização da complexidade em projetos de engenharia. Gestão da Produção, 25(2), 331-342. https://doi.org/10.1590/0104-530X2957-16

Martins, A. R. Q.; Signori, G. G.; Capellari, M. R. S.; Sotille, S. S. \& Kalil, F. (2016). Uso de design thinking como experiência de prototipação de ideias no ensino superior. Future Studies Research Journal: Trends and Strategies, 8 (1), 208-224. https://doi.org/10.7444/future.v8i1.227

Maximiano, A. C. A. (2019). Administração por competências: você gestor. São Paulo, Brasil: Editora Atlas.

Menezes, U. G.; Kneipp, J. M.; Barbieri, L. A. \& Gomes, C. M. (2011). Gestão da inovação para o desenvolvimento sustentável: comportamento e reflexões sobre a indústria química. Revista de Administração e Inovação, 8 (4), 88-116. https://doi.org/10.5773/rai.v8i4.660

Moraes, M. O.; Glória Jr, I.; Neto, P. L. O. C. \& Gonçalves, R. G. (2017). Análise de um projeto de inovação tecnológica e o uso da abordagem Canvas. Iberoamerican Journal of Project Management, 8 (1), 14-26.

Oliveira, A. C. A. (2014). A contribuição do design thinking na educação. E-Tech: Tecnologias para Competitividade Industrial, 105-121. https://doi.org/10.18624/e-tech.v0i0.454

Pacheco, E. (2012). Perspectivas da educação profissional técnica de nível médio: proposta de diretrizes curriculares nacionais. São Paulo, Brasil: Editora Moderna.

Parolin, S. R. H. (2008). Elaboração de projetos inovadores na educação profissional. Curitiba, Brasil: SESI/SENAI/PR.

Peinado, J. \& Graeml, A. R. (2007). Administração da produção: operações industriais e de serviços. Curitiba, Brasil: UnicenP. 
Research, Society and Development, v. 11, n. 1, e5411124554, 2022

(CC BY 4.0) | ISSN 2525-3409 | DOI: http://dx.doi.org/10.33448/rsd-v11i1.24554

Project Management Institute (PMI). (2017). Um guia do conjunto de conhecimento em gerenciamento de projetos. 6ed. USA: Project Management Institute.

Ritter, B. A.; Small, E. E.; Mortimer, J. W. \& Doll, J.L. (2018). Designing management curriculum for workplace readiness: developing students soft skills. Journal of Management Education, 42 (1), 80-103. https://doi.org/10.1177/1052562917703679

Robles,M. M. (2012). Executive perceptions of the top 10 soft skills needed in today's workplace. Business Communication Quarterly, 75 (4), 453-465. https://doi.org/10.1177/1080569912460400

Rosa, C. A. (2014). Modelo de negócios: kit de ferramentas. Belo Horizonte, Brasil: SEBRAE/MG.

Silva Filho, A. M. (2015). Sobre a análise SWOT para planejamento e gestão de projetos. Revista Espaço Acadêmico, 169, 53-57. https://periodicos.uem.br/ojs/index.php/EspacoAcademico/article/view/28083

Sköldberg, U. J.; Woodilla, J. \& Çetinkaya, M. 2013. Design Thinking: past, present and possible futures. Creativity and Innovation Management, 22 (2), 121146. https://doi.org/10.1111/caim.12023 